\title{
Tuning photonic bandgap of three dimensional opal structures by utilizing polymer and silica hybrid colloids
}

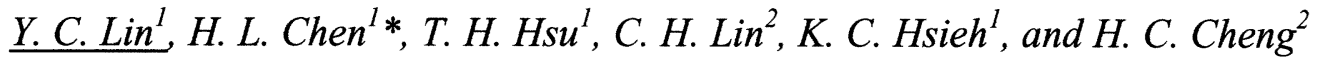 \\ ${ }^{I}$ Department of Materials Science and Engineering, National Taiwan University, Taipei, Taiwan \\ ${ }^{2}$ National Nano Device Lab., Hsinchu, Taiwan
}

Tel: 886-2-33663240, Fax: 886-2-23634562,*E-mail: hsuenlichen@ntu.edu.tw

Photonic crystals, which are the artificial multidimensional periodic structures, are attractive for ultra-compact optoelectronic devices. Various photonic crystals devices based on their photonic bandgap (PBG) have been reported. Recently, several methods have been developed for fabricating three-dimensional (3D) photonic crystals. One of the simple methods is using colloidal spheres such as polystyrene or silica beads to form 3D face-centered cubic crystalline structures. An important issue for colloidal spheres based PBG devices is how to fabricate the devices with small bandgap-shift for optoelectronic device applications. In general, it is difficult to tune the size of colloidal spheres continuously expecting for controlling the strict synthesis conditions $[1,2]$.

Generally the colloidal spheres based PBG structures are formed by the monodispersed silica or polystyrene beads, and their bandgaps are decided by the refractive index and diameter of beads. In this paper, we demonstrate a simple method that can control the bandgap position by utilizing hybrid polymer and silica beads with large size-difference. The polystyrene (PS) beads with $750 \mathrm{~nm}$ diameter and silica beads with $50 \mathrm{~nm}$ diameter are mixed to form different PBG structures. Figure 1 shows the SEM images of crystalline opal structures constructed by polystyrene beads with and without silica beads. We found that the polystyrene beads were separated by silica beads with $50 \mathrm{~nm}$ distance. As shown in Figure 2, the transmittance dips (bandgap) of opal structures were shifted continuously from $1730 \mathrm{~nm}$ to $1800 \mathrm{~nm}$ as increasing the silica colloid concentrations. The bandgap-shift percentages are also increased from $0.5 \%$ to $3.75 \%$. Moreover, we can study the effect of the distribution of silica beads among the polystyrene beads. The polystyrene beads are separated by $50 \mathrm{~nm}$ and hence the atomic packing factor (APF) degrades from $74 \%$ to $61 \%$. Figure 3 (a) and 3 (b) show the interstitial between separated polystyrene beads are filled with air hole and closed-packed silica beads, respectively. According to the Bragg law, the band-gap is shift to long wavelength with $1.708 \%$ for separating by air hole as shown in Table I. Similarly if the interstitial between separated polystyrene beads is filled with closed-packed silica beads. The band-gap will shift to long wavelength about $10.27 \%$ as shown in Table I. Comparing with our experimental results, we can propose that the content of silica beads of $10 \mu 1$ and $20 \mu 1$, the PS beads nearly maintains close-packed, but only a few silica beads locate in the interstitial of the PS beads. As the content of silica beads increased to $30 \mu 1,40 \mu 1$ and $50 \mu 1$, the probability of silica beads filled in the gap of polystyrene beads is increased, and the bandgap is shifted to longer wavelength. Detailed analysis will be reported in the conference.

\section{References}

1. J. Nestor, J. Esquena, C. Solans, B. Levecke, K. Booten and T. F. Tadros, Langmuir, 21, 4837, 2005

2. Z. S. Xu, Z. W. Deng, X. X. Hu, L. Li and C. F. Yi, Journal of Polymer Science-Part A : polymer chemistry, 43, 2368, 2005 


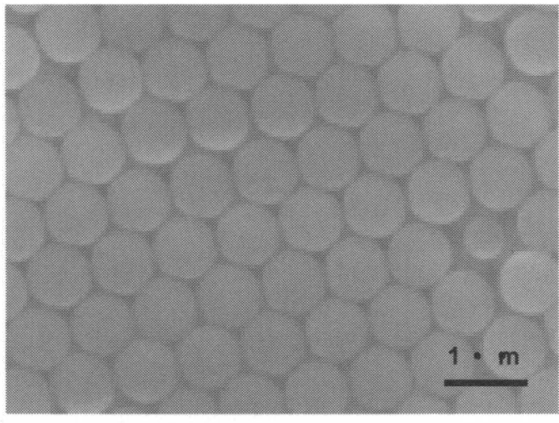

(a)

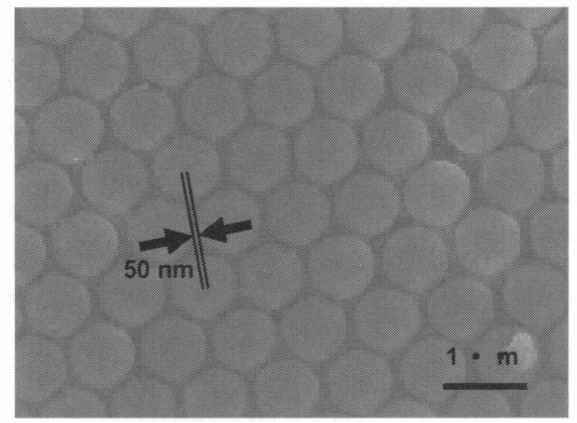

(b)

Fig. 1 SEM images of crystalline opal structures constructed by polystyrene beads (a) without (b) with silica beads

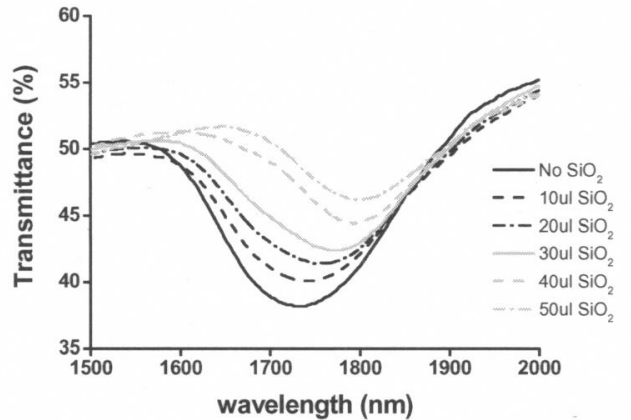

(a)

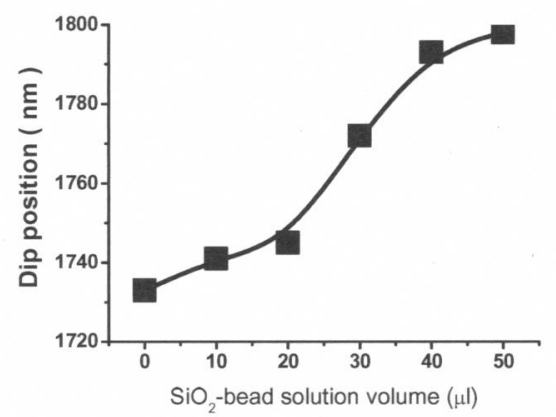

(b)

Fig. 2 (a) Transmission spectra and (b) bandgap position of polystyrene opal structures mixed by silica beads with various silica colloid concentrations.

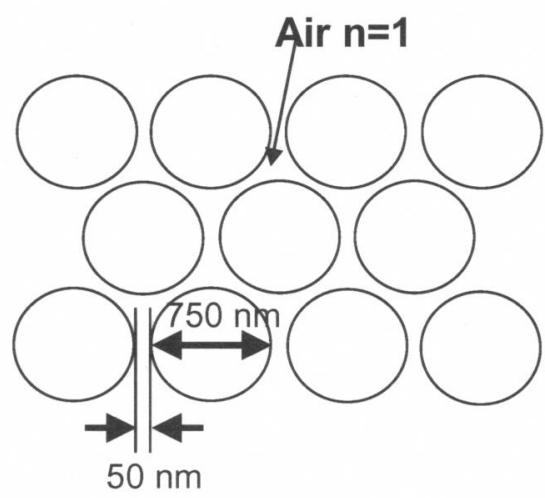

(a)

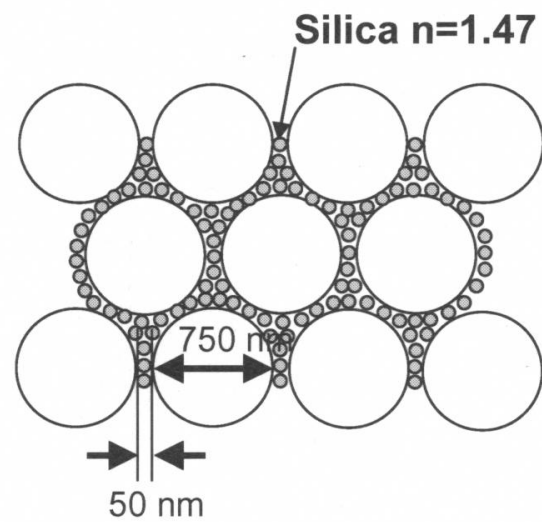

(b)

Fig. 3 The schematic diagrams of the interstitial polystyrene opal structures (a) polystyrene beads are separated by air-holes. (b) polystyrene beads are separated by close-packed silica beads.

Table I The different band-gap position are simulated by Bragg law

\begin{tabular}{|c|c|c|c|}
\hline & $\begin{array}{c}\text { Close-packed } \\
\text { PS beads }\end{array}$ & $\begin{array}{c}\text { PS beads separated } \\
\text { by air-hole }\end{array}$ & $\begin{array}{c}\text { PS beads separated by } \\
\text { silica beads }\end{array}$ \\
\hline Band-gap position & $1768.6 \mathrm{~nm}$ & $1797.8 \mathrm{~nm}$ & $1950.3 \mathrm{~nm}$ \\
\hline Band-gap shift percentage (\%) & $0 \%$ & $1.708 \%$ & $10.27 \%$ \\
\hline
\end{tabular}

